\title{
The Cochaperone BAG2 Sweeps Paired Helical Filament- Insoluble Tau from the Microtubule
}

\author{
Daniel C. Carrettiero, ${ }^{1,3 *}$ Israel Hernandez, ${ }^{1 *}$ Pierre Neveu, ${ }^{1,2}$ Thales Papagiannakopoulos, ${ }^{1}$ and Kenneth S. Kosik ${ }^{1}$ \\ ${ }^{1}$ Neuroscience Research Institute, and ${ }^{2}$ Kavli Institute for Theoretical Physics, University of California Santa Barbara, Santa Barbara, California 93106, and \\ ${ }^{3}$ Biosciences Institute, Department of Physiology, University of Sao Paulo, 05508-090, Sao Paulo, Brazil
}

\begin{abstract}
Tau inclusions are a prominent feature of many neurodegenerative diseases including Alzheimer's disease. Their accumulation in neurons as ubiquitinated filaments suggests a failure in the degradation limb of the Tau pathway. The components of a Tau protein triage system consisting of CHIP/Hsp70 and other chaperones have begun to emerge. However, the site of triage and the master regulatory elements are unknown. Here, we report an elegant mechanism of Tau degradation involving the cochaperone BAG2. The BAG2/Hsp70 complex is tethered to the microtubule and this complex can capture and deliver Tau to the proteasome for ubiquitin-independent degradation. This complex preferentially degrades Sarkosyl insoluble Tau and phosphorylated Tau. BAG2 levels in cells are under the physiological control of the microRNA miR-128a, which can tune paired helical filament Tau levels in neurons. Thus, we propose that ubiquitinated Tau inclusions arise due to shunting of Tau degradation toward a less efficient ubiquitin-dependent pathway.
\end{abstract}

Key words: BCL-associated anthanogene2; BAG2; heat shock protein 70; Hsp 70; phosphorylated Tau; ubiquitin; proteasome; mir-128a

\section{Introduction}

Tau inclusions are classic hallmarks of many neurodegenerative diseases in which phosphorylated Tau proteins self-assemble into massive polymeric fibrils that are held together in an unknown linkage and resist the normal degradation mechanisms of the cell. Tau inclusions are ubiquitinated, both in poly ubiquitinated (Cripps et al., 2006) and mono-ubiquitinated linkages (Iqbal and Grundke-Iqbal, 1991; Morishima-Kawashima et al., 1993; Ii et al., 1997). The presence of ubiquitinated inclusions points to a defect in Tau protein triage, a single molecule decision system that assesses each Tau protein and decides whether the protein is functional, or misfolded and salvageable, or beyond repair and subject to proteolysis. Currently, the site of Tau triage is unknown. In the Tau inclusion diseases, at a point beyond this early triage decision, the cell makes an ensemble decision regarding its continued survival in the face of the overall burden due to inclusions or toxic oligomers.

The Tau protein triage system uses a complex consisting of the E3 ligase CHIP (C terminus of Hsp70-interacting protein) in conjunction with several chaperones to refold Tau or direct it toward the degradation machinery (Petrucelli et al., 2004; Shimura et al., 2004). Thus, the Tau/CHIP/heat shock complex lies at a pivotal decision node directing the fate of each Tau mol-

\footnotetext{
Received Sept. 29, 2008; accepted Dec. 11, 2008.

This work was supported by the National Institutes of Health, the Hillblom Foundation, the Everett Fisher Foundation, and National Science Foundation Grant PHY05-51164. D.C.C. was a visiting fellow and received his fellowship from Coordenação de Aperfeiçoamento de Pessoal de Nível Superior (CAPES; BEX4494-05-9). We thank Stuart Feinstein and DeeAnn Hartung for their help with the pulse chase experiments.

The authors declare no competing financial interests.

${ }^{*} D$.C.C. and I.H. contributed equally to this work.

Correspondence should be addressed to Kenneth S. Kosik at the above address. E-mail: kosik@lifesci.ucsb.edu. DOI:10.1523/JNEUROSCI.4660-08.2009

Copyright $\odot 2009$ Society for Neuroscience $\quad$ 0270-6474/09/292151-11\$15.00/0
}

ecule recognized by the complex toward degradation or restitution. The accumulation of ubiquitinated Tau proteins in cells could arise due to the blockade of a ubiquitin-dependent Tau degradation pathway or inappropriate shuttling to the ubiquitindependent pathway from a preferred route. Indeed, other Tau degradation pathways have been described including ubiquitinindependent Tau degradation and caspase-mediated degradation (Berry et al., 2003; Ding et al., 2006).

The observation that the CHIP/Hsp70 complex specifically ubiquitinates AD-type hyperphosphorylated Tau (p-Tau) suggested that phosphorylation is the signal for at least one Tau ubiquitination pathway (Shimura et al., 2004). Increasing Hsp70 reduced total Tau levels and attenuated Tau aggregation suggesting that the CHIP/Hsp70 can promote Tau degradation (Petrucelli et al., 2004). In vivo evidence supports the attenuation of Tau aggregation by CHIP upregulation (Sahara et al., 2005). Small molecule inhibition of Hsp90, which binds the Hsp70/substrate complex, can decrease p-Tau in a mouse tauopathy model (Dickey et al., 2007).

Our findings reveal a novel and highly efficient pathway of Tau degradation that operates in proximity to the microtubule, is ubiquitin-independent, and is regulated by miR-128, a microRNA that is increased in Alzheimer's disease (Lukiw, 2007). This pathway is mediated by the cochaperone BAG2. Members of the BAG family interact with the ATPase domain of Hsp70 through their BAG domains (Takayama et al., 1999) and stimulate the degradation of the chaperone clients in the proteasome. In the case of BAG1, degradation of the glucocorticoid hormone receptor (Demand et al., 2001) occurs in a ubiquitin-dependent manner via the BAG1 ubiquitin-like domain. However, BAG2 lacks the ubiquitin-like domain (Lüders et al., 2000; Alberti et al., 2002), and therefore, may be suited to triage client proteins independently of ubiquitin. 


\section{Materials and Methods}

Antibodies, reagents and plasmids. The following antibodies were used for immunoblotting and/or immunofluorescence: TAU-5 antibody (1:1000, Biosource), which recognizes phosphorylated and nonphosphorylated forms of Tau. Phosphorylation-dependent Tau antibodies included paired helical filament-1 (PHF-1) monoclonal antibody, which recognizes Ser-396 and Ser-404 residues (1:500; provided by P. Davies, Feinstein Institute for Medical Research, Manhasset, NY); T181 monoclonal antibody (1:1000, Sigma); S199/202 rabbit monoclonal antibody (1:1000, Sigma). Also used were Flag antibody (1:1000, Sigma); rabbit polyclonal BAG2 antibody (1:500, Abcam, clone ab58682); mouse anti- $\alpha$ tubulin (1:50, Sigma), mouse mono- and polyubiquitinylated proteins (1:20, clone FK2, BIOMOL), mouse $\beta$-actin monoclonal (1:10,000, Sigma), rabbit anti-CHIP (N terminal) (Sigma-Aldrich, C9118), and mouse anti-Hsp70/Hsc70 mAb (Stressgen, BB70). Lactacystin, a proteasome inhibitor (Fenteany and Schreiber, 1998), was used at $10 \mu \mathrm{M}$ (Calbiochem). Benzyloxycarbonyl-valinyl-alaninyl-aspartyl fluoromethyl ketone (Z-VAD.FMK), an interleukin- $1 \beta$-converting enzyme-like protease inhibitor, was used at $20 \mu \mathrm{M}$.

Human 4R Tau and mouse BAG2-Flag cDNAs were cloned into pEYFP-C1, pDsRed2-C1 and pECFP-C1 vectors (Clontech). The RNAi sequences were obtained by running an algorithm for picking siRNA sites (Heale et al., 2005) and cloned into pSilencer 4.1-CMV puro vector (Ambion). The negative control construct was altered so that the sequence was no longer complementary to BAG2 mRNA. The BAG2 shRNAi sequences synthesized were: Sense strand GCCGGACCCUCACGGUUGAgg and antisense strand UCAACCGUGAGGGUCCGGCcc (overhang in lower case). The wild-type human Ubiquitin C expression plasmid (\#11928) and the Ub-KO plasmid with all seven lysines of ubiquitin mutated to arginines (plasmid \#11934) were purchased from Addgene (Bergink et al., 2006; Dantuma et al., 2006). The K48R ubiquitin mutant was prepared by site-directed mutagenesis on a plasmid expressing mVenus-UBB (Quick-Change II site-directed mutagenesis Kit, Stratagene). Forward primer 5 '-gctcatctttgcaggccggcagctggaagatggc, and reverse primer $5^{\prime}$-gccatcttccagctgccggcctgcaaagatgagc were used to introduce a lysine codon (aag) for an arginine codon (cgg) at position 48 of the ubiquitin protein. The mutagenesis was confirmed by sequencing using the primer $5^{\prime}$-cttaccggcaagaccatc.

Cell culture. Monkey kidney COS-7 cells were grown in DMEM supplemented with $10 \%$ fetal bovine serum and penicillin/streptomycin (Invitrogen) in a $5 \% \mathrm{CO}_{2}$ humidified incubator at $37^{\circ} \mathrm{C}$. Cells were transfected with Lipofectamine (Invitrogen) and lysed with RIPA buffer (1\% Triton X-100, 0.5\% sodium deoxycholate, $0.1 \%$ SDS, $150 \mathrm{~mm} \mathrm{NaCl}, 50$ $\mathrm{mm}$ TrisHCl, $\mathrm{pH}$ 7.4). Protein concentration was estimated by the BCA protein assay kit (Pierce) and was adjusted to $1 \mu \mathrm{g} / \mu \mathrm{l}$.

Pregnant embryonic day 18 (E18) Sprague Dawley rats were killed by $\mathrm{CO}_{2}$ incubation, and embryos were removed immediately by Cesarean section. Hippocampi were removed in dissection media without calcium and magnesium (HEPES buffered HBSS; HEPES, $10 \mathrm{~mm}, \mathrm{pH} 7.3$, and Pen/Strep) and digested in $0.25 \%$ trypsin with the same dissection media at $37^{\circ} \mathrm{C}$ for $15 \mathrm{~min}$. The tissue was then washed $2 \times$ with HBSS and manually dissociated with a fire-bored Pasteur pipette. Cells were plated at 250,000 cells per six-well plate for immunoblot analyses and 90,000 cells per six-well plate for immunofluorescence. The plates were previously coated overnight with poly-L-lysine and incubated with glial medium (MEM, 20\% glucose, pyruvate, Pen/Strep and 10\% horse serum) until plating. Three hours after plating, the medium was changed to Neurobasal medium containing B27 supplement and $0.5 \mathrm{~mm}$ glutamine. Very few glial cells were observed in these cultures.

Pulse-chase. COS7 cells were cotransfected with TAU and BAG2 or transfected with TAU in the absence of BAG2. Sixteen hours after transfection, cells were incubated for 30 min in DMEM methionine/cysteine Free, supplemented with dialized FBS and L-glutamine. Cells were incubated in the same media with Expre ${ }^{35} S^{35} S$ protein labeling mix (200 $\mu \mathrm{Ci} / \mathrm{ml}$; PerkinElmer) for $1 \mathrm{~h}$. After this pulse period, media was removed and cells were washed twice with prewarmed PBS and incubated up to $28 \mathrm{~h}$ in the same media with unlabeled L-cysteine- $\mathrm{HCl}(500 \mu \mathrm{g} / \mathrm{ml}$; Sigma-Aldrich) and L-methionine (100 $\mu \mathrm{g} / \mathrm{ml}$; Sigma-Aldrich). Cells were harvested at $0,4,8,24$, and $28 \mathrm{~h}$ after the pulse period. For harvesting, cells were washed once in prewarmed PBS, scraped from the well, centrifuged at $2300 \mathrm{rpm} / 5 \mathrm{~min} / 4^{\circ} \mathrm{C}$. The pellet of cells were resuspended in lysis buffer ( $150 \mathrm{~mm} \mathrm{KCl,} 25 \mathrm{~mm}$ TrisHCl, 2 mm EDTA, $0.5 \mathrm{~mm}$ DTT, $0.5 \% \mathrm{NP}-40$ and $1 \times$ protease inhibitor cocktail; Sigma-Aldrich), and incubated at $4^{\circ} \mathrm{C}$ for $30 \mathrm{~min}$, in a head-to-tail mixer. Lysates were centrifuged at $13,200 \mathrm{rpm} / 1 \mathrm{~min} / 4^{\circ} \mathrm{C}$ and the supernatant were transferred to a microcentrifuge tube. The lysates were immunoprecipitated with PHF-1 antibody, using protein-G Sepharose. Spin Column (Sigma-Aldrich) were used to minimize Sepharose beads lost during washing times. Immunoprecipitated $\mathrm{p}$-Tau was eluted using $1 \times$ Laemmli sample buffer and separated in an SDS-PAGE $10 \%$ gel $(1.0 \mathrm{~mm})$. Acrylamide gels were Coomassie-blue stained and then incubated for $30 \mathrm{~min}$ with EN3HANCE enhancer solution (PerkinElmer), washed on cold water with $1 \%$ glycerol and dried. A PhosphorImager screen was exposed for $3 \mathrm{~d}$ at $-80^{\circ} \mathrm{C}$ and scanned images were quantified with quantity-one software (Bio-Rad Laboratories)

Transfections, RNA isolation, qPCR. To overexpress Tau and BAG2 protein, COS-7 cells were transfected with $4 \mu \mathrm{g}$ of BAG2 RNAi or a nonsilencing RNAi vector in a six-well plate with Lipofectamine (Invitrogen). Forty-eight hours later, the cells were cotransfected with $2 \mu \mathrm{g}$ of $4 \mathrm{R}$ Tau (pDsRed2-C1) and $2 \mu \mathrm{g}$ of BAG2-Flag pEYFP-C1. Twentyfour hours later, cells were lysed for immunoblotting. All experiments in primary neurons were initiated $5 \mathrm{~d}$ after plating, and the amount of transfected plasmid was half of the amount used in COS-7 cells. To overexpress mir-128a, we transfected 250,000 neurons at day in vitro 5 (DIV5) with 75 or $250 \mathrm{~nm}$ pre-mir-128a or scrambled (Ambion) using Lipofectamine. For transfections, lysates were harvested $48 \mathrm{~h}$ later. For RNA isolation and qPCR, precipitates were added to cells and medium was renewed after $5 \mathrm{~h}$. After the indicated time, RNA was extracted by using the miRVana Isolation Kit (Ambion) and DNase treated (DNAfree, Ambion). Reverse transcription was performed by using Superscript III (Invitrogen). Quantitative real-time PCR was performed in an Applied Biosystems PRISM 7900HT fast real-time PCR System with SYBR green PCR master mix (Applied Biosystems). BAG2 Ct was normalized to that of GAPDH.

Dual luciferase assay. Downstream of the Firefly luciferase reporter vector pMIR-Report (Ambion) we inserted a $60 \mathrm{bp}$ sequence of the BAG2 3'UTR that contained the miR-128a predicted target site. Our negative control construct was altered so that three bases at the seed region were no longer complementary to miR-128a. We seeded 50,000-60,000 HeLa cells $24 \mathrm{~h}$ before transfection in 24-well tissue culture plates. The next day, $200 \mathrm{ng}$ of the pMIR-Report vector, $20 \mathrm{ng}$ of the transfection control Renilla vector phRLTK (Promega), and 30 pmol pre-mir-128a or scrambled were transfected using $3 \mu \mathrm{l}$ of lipofectamine. Lysates were harvested $24 \mathrm{~h}$ after transfection and reporter activity was measured using the Dual Luciferase Assay (Promega). The Firefly luciferase units from every sample were normalized to the units of the transfection control Renilla.

Preparation of Sarkosyl insoluble Tau. Preparations of Sarkosyl insoluble Tau were previously described (DeTure et al., 2002; Cho and Johnson, 2004). Briefly, cells were prepared in lysis buffer containing $50 \mathrm{~mm}$ TrisHCl, pH 7.4, $0.15 \mathrm{M} \mathrm{NaCl}$, and $1 \%$ Sarkosyl and scraped off the plate after $30 \mathrm{~min}$ incubation at $4^{\circ} \mathrm{C}$. The samples were vortexed, incubated for $30 \mathrm{~min}$ at RT, centrifuged $20 \mathrm{~min}$ at $3000 \times \mathrm{g}$. The supernatants were recovered and centrifuged at $170,000 \times g$ for $2 \mathrm{~h}$ at $4^{\circ} \mathrm{C}$. Recovered supernatants were mixed with electrophoresis sample buffer. Pellets containing Sarkosyl insoluble material were mixed with $2 \times$ sample buffer. All the samples were boiled at $100^{\circ} \mathrm{C}$ for $5 \mathrm{~min}$. Protein was separated on a $4-20 \%$ gradient SDS-PAGE, transferred to a Protran membrane, incubated with the relevant antibodies and detected with horseradish peroxidase conjugated antibodies. Bands were visualized with chemiluminescence (Pierce).

Immunoprecipitation. COS-7 cells were transfected with $4 \mu \mathrm{g}$ of each plasmid (BAG2 and Tau). Twenty-four hours later, cells were washed in cold PBS and harvested in immunoprecipitation buffer $(150 \mathrm{~mm} \mathrm{NaCl}$, 0.5\% Triton X-100, 50 mм TrisHCl, pH 7.5, 5 mм EDTA, 1 mм DTT, 1 $\mathrm{mM}$ PMSF and protease inhibitor mixture). The lysate was precleared for $1 \mathrm{~h}$ at $4^{\circ} \mathrm{C}$ with $25 \mu \mathrm{l}$ of protein $\mathrm{G}$ (Sigma) and centrifuged at 14,000 rpm. The supernatant was incubated with $5 \mu \mathrm{g}$ of antibody against TAU- 5 and 
$60 \mu \mathrm{l}$ of protein $\mathrm{G}$ and rocked at $4^{\circ} \mathrm{C}$ overnight. The protein $\mathrm{G}$ beads were pelleted and washed with immunoprecipitation buffer. The precipitates were resolved by SDS-PAGE gel and immunoblotted. $\beta$-Actin was used to normalize Tau loading. Statistical analysis were performed with PRISM Software (GraphPad software) using the Dunnett's multiple comparison test after one-way ANOVA.

Primary rat hippocampal neuronal cultures were infected at DIV 1 with BAG2-FLAG lentivirus or a GFP control lentivirus and grown for 1 week. The cells were then washed twice with prewarmed PBS and incubated for $30 \mathrm{~min}$ in lysis buffer $(150 \mathrm{~mm} \mathrm{KCl}, 25 \mathrm{~mm}$ TrisHCl, $2 \mathrm{~mm}$ EDTA, 0.5 mм DTT, $0.5 \%$ NP-40 and $1 \times$ Protease Inhibitor Cocktail (Sigma-Aldrich) at $4^{\circ} \mathrm{C}$. They were scraped from the well and the lysates were centrifuged at $13,200 \mathrm{rpm} / 10 \mathrm{~min} / 4^{\circ} \mathrm{C}$, and the supernatant was recovered. Approximately $600 \mu \mathrm{l}$ of lysate were immunoprecipitated with PHF-1 antibody or with anti-FLAG M2 agarose affinity gel (SigmaAldrich). Immunoprecipitates were eluted with $1 \times$ loading sample buffer and Western blot was performed with anti-FLAG, PHF-1, antiCHIP, anti-HSP70.

Immunohistochemistry. For immunofluorescent labeling, neurons or COS-7 cells were grown on glass coverslips and gently washed $(3 \times 1$ min) in warmed MEM-H (1.423\% of MEM with HEPES, $0.22 \%$ $\left.\mathrm{NaHCO}_{3}\right), \mathrm{pH}$ 7.2. The cells were fixed in $4 \%(\mathrm{w} / \mathrm{v})$ paraformaldehyde $\left(15 \mathrm{~min}, 37^{\circ} \mathrm{C}\right)$, washed $(3 \times 1 \mathrm{~min})$ with $\mathrm{PBS}$, permeabilized with $0.1 \%$ Triton X-100 (15 min), washed $2 \times$ in PBS and blocked for 20 min with blocking medium (MEM-H, 1\% BSA, 10\% horse serum). Cells were incubated with primary antibodies in blocking medium for $2 \mathrm{~h}$ at room temperature, washed $(3 \times 5 \mathrm{~min})$ with PBS and incubated for an additional $1 \mathrm{~h}$ with secondary antibodies. Finally, cells were washed in PBS and visualized by fluorescence microscopy (Nikon eclipse TE300).

To analyze the effect of BAG2 overexpression, BAG2 RNAi overexpression or BAG2 RNAi nonsilencing on Tau levels, BAG2-pEYFP-C1 vector, BAG2RNAi plus pEYFP-C1 vector and BAG2 RNAi nonsilencing-pEYFP-C1 vector were separately transfected into 5-d-old neurons plated on glass coverslips. After $3 \mathrm{~d}$, neurons were fixed and labeled with antibody. In each transfection experiment, 100 BAG2 positive and 100 BAG2 negative neurons were randomly chosen in the same plate. Optical density (OD) values of PHF-1 stained neurons were compared among the transfected and nontransfected neurons in the same plate. Quantification of pixel intensity was performed with MetaMorph software and normalized to background. The number of cells in each group with $\mathrm{OD}>200$ and $\mathrm{OD}<200$ were binned.

For colocalization studies, COS-7 cells were transfected with BAG2 and Tau for $24 \mathrm{~h}$, fixed in $4 \%$ paraformaldehyde, and stained with mouse monoclonal anti- $\alpha$-tubulin and counterstained with Alexa 594 antibody. Data were acquired on a spinning disc microscope (Olympus) and then postprocessed by ImageJ software (http://rsb.info.nih.gov/ij/). Images were processed using successive median and Wiener filtering to reduce noise.

BAG2 mobility assay. COS-7 cells were transfected with BAG2pEYFP-C1 plasmid and/or with Tau-pECFP-C1 plasmid in 1:4 ratio. Six hours after transfection, $100 \mu \mathrm{M}$ 3,4-methylenedioxy- $\gamma$-benzylidine- $\gamma$ butyrolactam (KNK437) was added. After $20 \mathrm{~h}$, the cells were imaged on an Olympus IX50 inverted microscope with an Olympus UPLSAPO $60 \times$ $\mathrm{NA}=1.2$ water-immersion objective and a DVC-1310 CCD camera (DVC Company). With the 8-bit camera used for this study, gain was set to avoid saturated pixels in the image. Images were analyzed with custom-written Matlab (Mathworks) programs to extract puncta trajectories. Images were first segmented to extract the puncta, their center was determined by adjusting the background- and intensity-corrected intensity profile to a Gaussian curve. Puncta trajectories were then determined by matching puncta positions between frames using ascending pair wise distances and using puncta diameter and intensity as criteria to confirm the match. See supplemental data, available at www.jneurosci.org as supplemental material, for additional details.

\section{Results}

\section{BAG2 preferentially decreases levels of insoluble Tau and} phosphorylated Tau

Insoluble Tau is operationally defined as the fraction of Tau that pellets in the presence of the detergent Sarkosyl. When the levels of this insoluble pool of Tau are increased, neurons are predisposed to neurofibrillary tangle formation (Ballatore et al., 2007). The overexpression of BAG2 in primary neurons and the expression of BAG2 and Tau in COS-7 cells both resulted in a marked and selective decrease in the Sarkosyl insoluble Tau fraction as detected by two Tau antibodies: TAU-5 and PHF-1 (Fig. 1A). TAU-5 recognizes total Tau and PHF-1 recognizes a form of phosphorylated Tau thought to be vulnerable to misfolding.

COS-7 cells were used to investigate the effects of BAG2 on phosphorylated and nonphosphorylated Tau levels (Fig. 1 B; supplemental data, available at www.jneurosci.org as supplemental material). Phosphorylated Tau was detected with PHF-1 (detects Ser-396 and Ser-404, Jicha et al., 1999), Tau181 (Vanmechelen et al., 2000), and S199/202 (Jenkins et al., 2000), and total Tau, both phosphorylated and nonphosphorylated isoforms, was detected with TAU-5 (Berger et al., 2006). Transfection of BAG2 markedly decreased the levels of exogenous phosphorylated Tau in COS-7 cells as detected by these three different phospho-tau markers (Fig. 1 B). The change in PHF-1, T181 and S199/202 immunoreactivity compared with TAU-5 suggested that the predominant effect of BAG-2 was on phosphorylated Tau. While total Tau was reduced only $40 \%$, we observed a 3 - to 4 -fold decrease for PHF-1, a 10- to 12-fold decrease was observed for Tau181 and an 8- to 10 -fold decrease for S199/200. Thus, the degradation rate of phosphorylated Tau induced by BAG-2 is at least four times larger than total Tau, a sufficiently large difference that all phospho-Tau can be degraded without significantly affecting the pool of nonphospho-Tau. The effects of BAG2 overexpression on Tau levels were reversed in the presence of BAG2 RNAi (Fig. $1 B$ ). Knock down of BAG2-Flag protein was confirmed by immunoblot (Fig. 1B).

Pulse-chase experiments were conducted to test whether the effect of BAG2 on Tau was due to enhanced degradation or decreased synthesis (Fig. 1D; supplemental data, available at www. jneurosci.org as supplemental material). When cotransfected with BAG2, Tau had a half-life of $3 \mathrm{~h}$, which corresponds to a degradation rate of $0.23 \pm 0.04 \mathrm{~h}^{-1}$; whereas in the absence of BAG2, Tau had a half life of $20 \mathrm{~h}$, which corresponds to a degradation rate of $0.035 \pm 0.017 \mathrm{~h}^{-1}$. These control rates approximate values in the literature (David DC et al., 2002). Using Welch's $t$ test we calculated this change to be statistically significant $(p<0.001)$. The enhanced degradation of Tau was not accompanied by any change in Tau synthesis.

In neurons, overexpression of BAG2 also decreased the endogenous levels of phosphorylated Tau. These biochemical results were confirmed by immunocytochemistry (Fig. 1C). Transfection of BAG2-pEYFP-C1 into neurons decreased PHF-1 signal compared with BAG2 negative neurons. Out of 100 BAG2 positive neurons analyzed, $13 \pm 11 \%$ had an OD >200. BAG2pEYFP-C1 negative neurons in the same plate showed the opposite result: $92 \pm 6 \%$ of neurons had an OD $>200$. Using the same quantification for the experimental control, empty vector pEYFP-C1 did not change PHF-1 immunoreactivity. Analysis of the neurons by immunoblot gave results that were consistent with the immunocytochemistry (Fig. 1C). The decrement in Tau in neurons following BAG2 expression affected both the phosphorylated and total pools of Tau similarly (Fig. 1C) probably because phosphorylated Tau represents a greater proportion of total Tau in neurons.

BAG2 RNAi was used to suppress endogenous BAG2 in neurons. This treatment increased the levels of endogenous PHF-1 immunoreactivity by Western blot (Fig. $1 B$ ) and by immunohistochemistry (Fig. 1C). Out of 100 neurons, the number of neu- 
A
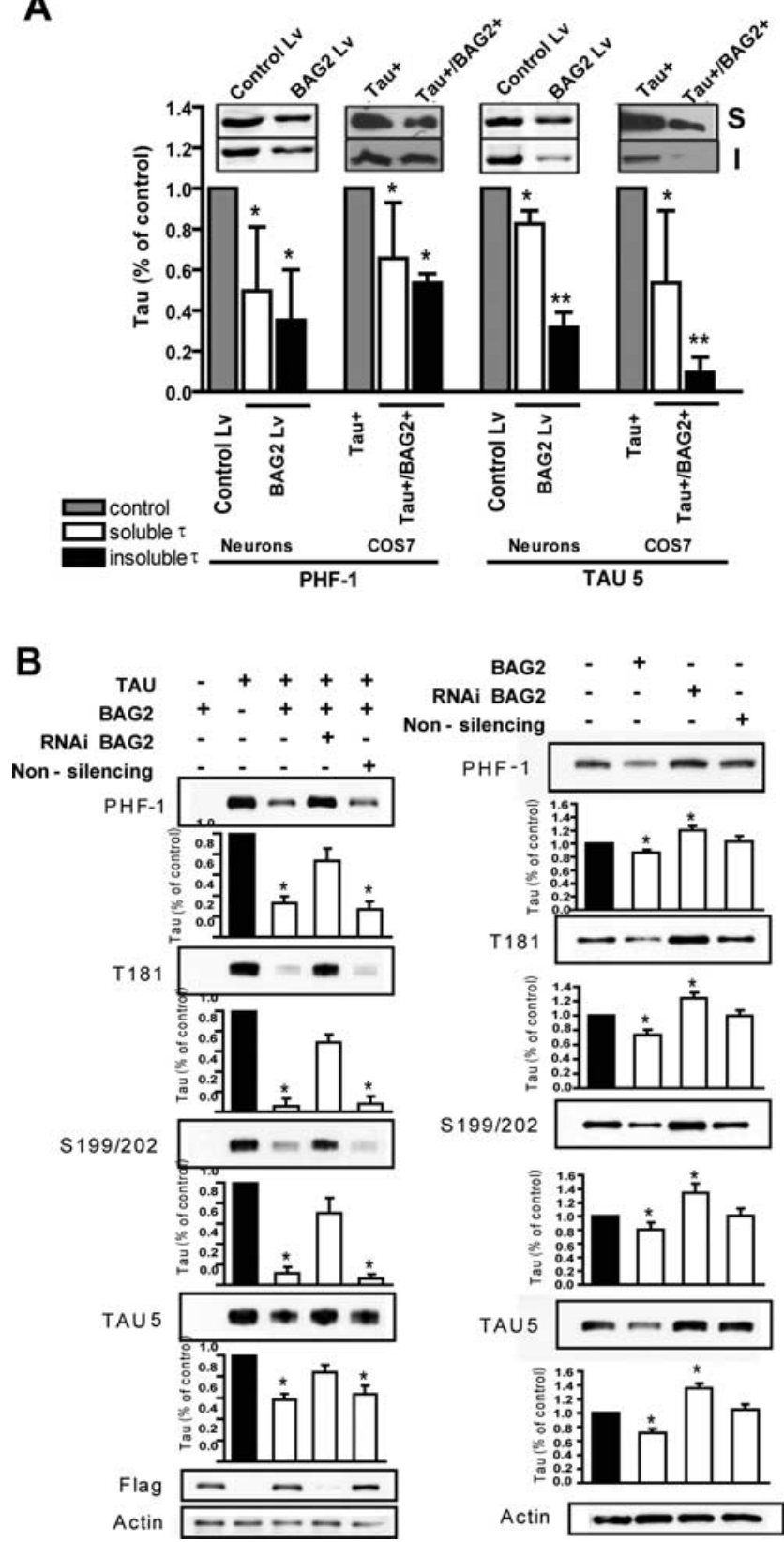

C
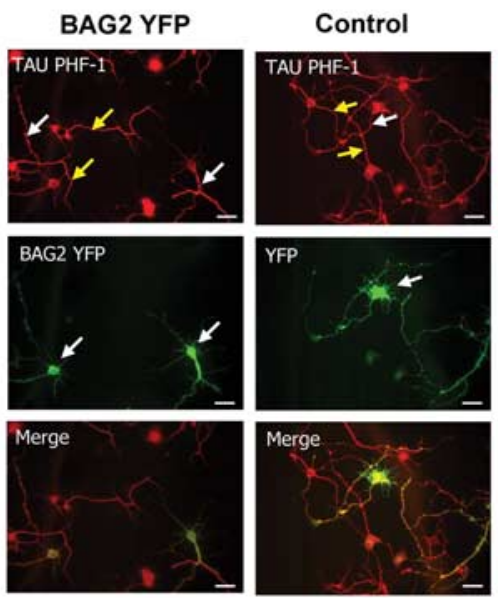

RNAi
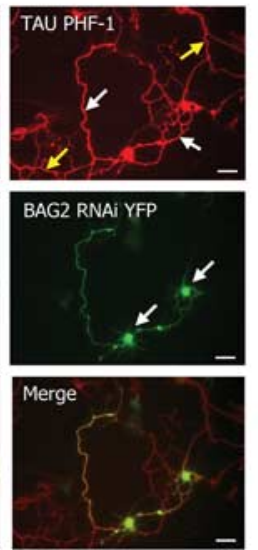

D

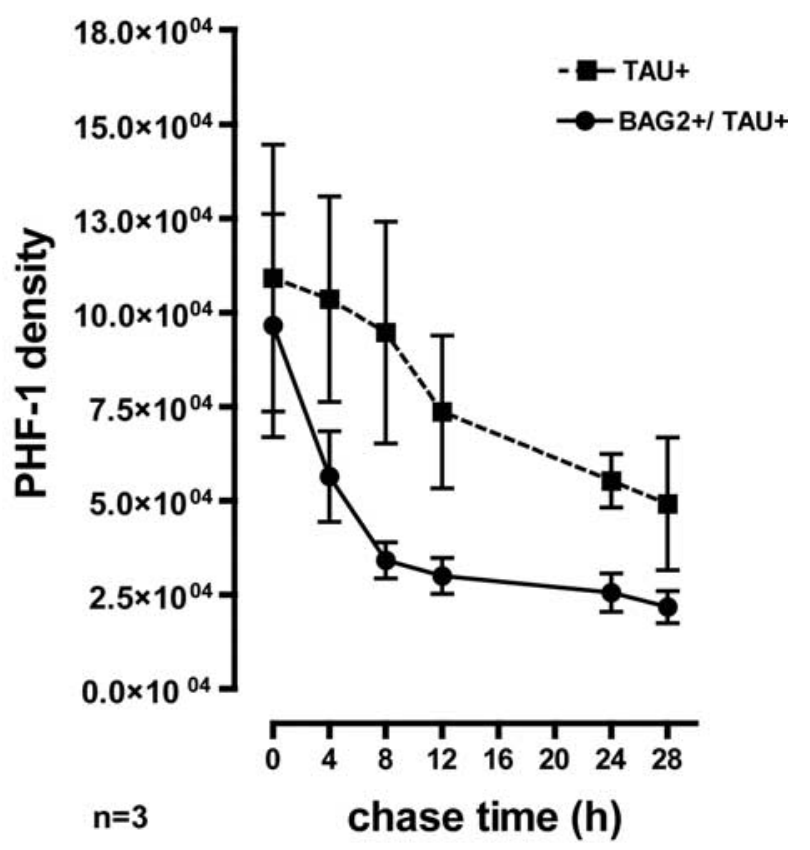

Figure 1. BAG2 reduces Tau levels. A, Neurons were transduced with a Lentivirus at DIV 1 containing a BAG2 RNAi sequence or a Lentivirus containing BAG2. After $8 \mathrm{~d}$, the neurons were fractionated into Sarkosyl soluble and insoluble pools and Tau was detected with PHF-1 and TAU-5 antibodies. COS7 cells were transfected with Tau and BAG2 and $24 \mathrm{~h}$ later Sarkosyl soluble and insoluble fractions were purified and detected with PHF-1 and TAU5 antibodies. Blots were quantified by densitometry using ImageJ and the values were normalized to the density of the control experiment (in the absence of BAG2). B, BAG2 overexpression decreased phosphorylated Tau. COS7 cells were transfected as indicated with Tau, BAG2 and BAG2 RNAi for $24 \mathrm{~h}$. Phosphorylated Tau was detected with PHF-1, T181 and S199/202. Total Tau was detected with TAU-5. BAG2 RNAi restored Tau levels. Flag antibody was used to detect overexpression of BAG2-Flag. Bands were quantified as a percentage of the control (black bar, left). Hippocampal cultured neurons were transfected to express BAG2-pEYFP-C1, BAG2 RNAi pEYFP, or nonsilencing pEYFP-C1. BAG2 induced a significant decrease in endogenous Tau levels as shown by immunoblot with Tau antibodies (PHF-1, T181, S199/202 and TAU-5) and conversely BAG2 RNAi increased Tau immunoreactivity with the same antibodies. Bands were quantified as a percentage of the control (black bar, right). Actin was used as a loading control (right). $\left({ }^{*} p<0.05\right.$, one-way ANOVA and Dunnett's multiple comparison test). Values are shown as \pm SEM, $n=3$. C, BAG2 levels regulate endogenous Tau in primary hippocampal neurons. Effects of BAG2-pEYFP-C1 (green) or BAG2 RNAi pEYFP-C1 (green) on PHF-1 immunoreactivity (red) in hippocampal neurons (white arrows: cells transfected with a pEYFP-C1 construct; yellow arrows: nontransfected cells). Scale bar, $20 \mu \mathrm{m}$. D, COS7 cells were either transfected with Tau or cotransfected with Tau and BAG2 for $16 \mathrm{~h}$; then cells were incubated in the presence of $\left[{ }^{35} \mathrm{~S}\right] \mathrm{Met} /\left[{ }^{35} \mathrm{~S}\right] \mathrm{Cys}$ mixture for $1 \mathrm{~h}$ and incubated up to $28 \mathrm{~h}$ in DMEM in an excess of nonlabeled L-methionine and L-cysteine-HCl. Cells were lysed at different chase times and pTau was immunoprecipitated and separated in SDS-PAGE. Radioactivity was detected by exposing the Phosphorlmager screen and densitometry of the signal was quantified.

rons with PHF-1 staining above an OD of 200 was increased by $1.7 \pm 0.3$-fold. Overexpression of pEYFP-C1 nonsilencing did not change the OD values with PHF-1 when compared with nontransfected neurons on the same plate (data not shown).
BAG2-mediated degradation of Tau is ubiquitin independent The association of BAG2 and Tau was validated by their coimmunoprecipitation. Primary neurons from rat hippocampus or cortex were infected with BAG2-Flag Lentivirus $(+)$ or an empty 
A

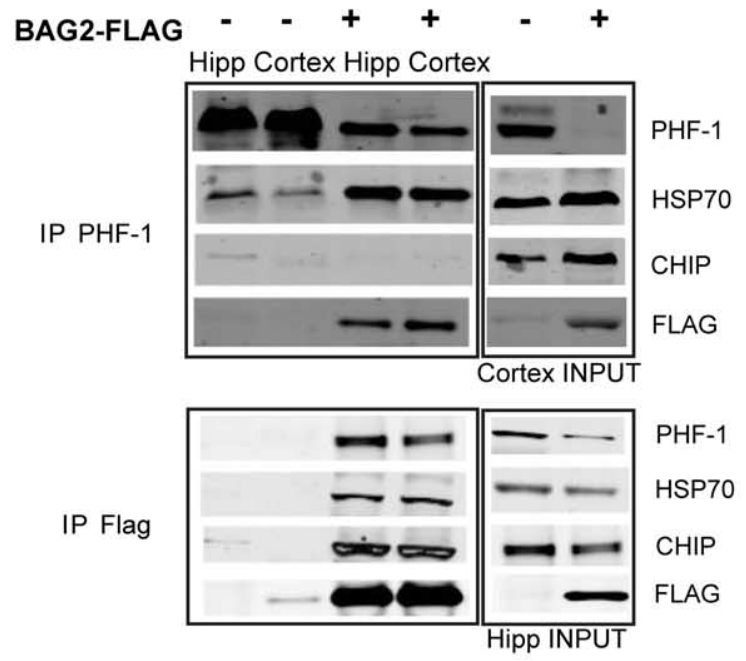

B

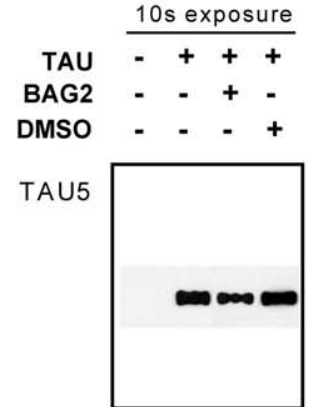

Tau 5

\section{$15 \mathrm{~min}$ exposure}

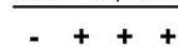

$-++$
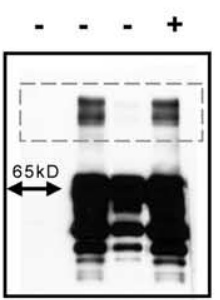

Tau5

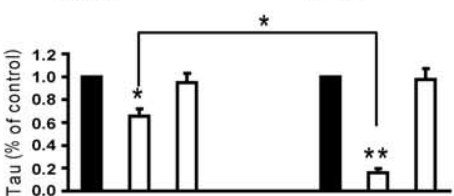

in

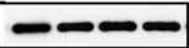

D
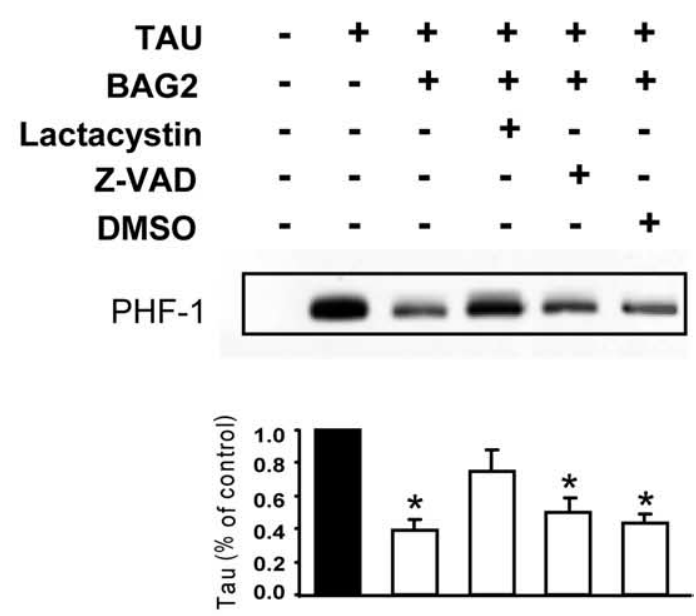

TAU 5
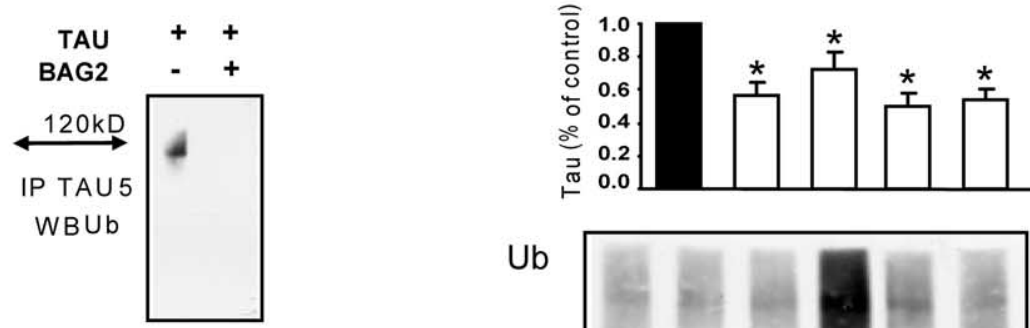

Ub

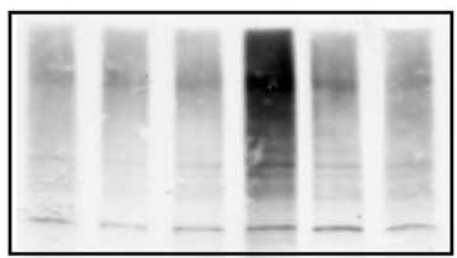

Actin

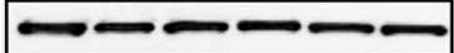

C

$\begin{array}{rllllllllll}\text { TAU } & - & + & + & + & + & + & + & + & + \\ \text { BAG2 } & - & - & + & + & + & + & - & - & - \\ \text { Ub K48R } & - & - & - & + & - & - & + & - & - \\ \text { Ub KO } & - & - & - & - & + & - & - & + & - \\ \text { Ub WT } & - & - & - & - & - & + & - & - & + \\ \text { PHF-1 }\end{array}$

Figure 2. BAG2 directs Tau to a Ubiquitin independent pathway. A, BAG2 and PHF-1 coimmunoprecipitate with Hsp70. Primary neuronal rat cultures from hippocampus (HIP) or cortex were infected with BAG2-Flag Lentivirus (+) or an empty Lentivirus ( - ) at DIV 1 and incubated for 1 week. Cells were lysed and lysates were immunoprecipitated with PHF-1 (top blot) or M2-Agarose Flag (bottom blot). BAG2 and PHF-1 coimmunoprecipitated and HSP70 was detected in the PHF-1/BAG2 complex. B, BAG2 inhibits ubiquitination of tau. Ubiquitinated Tau bands become apparent on a longer exposure blot with TAU-5 (compare 10 s to 15 min exposure). The high molecular weight ubiquitinated Tau bands are nearly eliminated in presence of BAG2 overexpression (top right arrow). The bands were quantified as a percentage of the control (black bar) $\left({ }^{*} p<0.05\right)$. To prove that the top band is ubiquitinated Tau, the samples were immunoprecipitated with TAU- 5 and blotted with a ubiquitin antibody (right panel). The ubiquitin-immunoreactive band comigrated with the high molecular weight band labeled with TAU-5. C, Dominant negative Ubiquitin mutants do not impair BAG2-mediated Tau degradation. COS7 cells were transfected with Tau and BAG2 in the presence of the Ubiquitin mutants, Ub-K48R, Ub-K0, or Ub WT. Twenty-four hours after transfection, lysates were blotted with PHF-1 or TAU-5. Actin was used as a loading control. D, Proteasome inhibition blocks the effects of BAG2. COS-7 cells were transfected with Tau and BAG2 and treated with the proteasomal inhibitor, lactacystin and the caspase inhibitor, Z-VAD in the combinations shown. PHF-1, TAU-5 and Ubiquitin antibodies were used to analyze the samples. Lactacystin blocked the effects of BAG2. The efficacy of lactacystin is shown by the increased ubiquitination in the bottom. The bands were quantified as percentage of the control (Tau in the absence of BAG2). ${ }^{*} p<0.05$. Statistical analyses were performed using one-way ANOVA followed by Dunnett's multiple comparison test. Student $t$ test was used to compare the effect of BAG2 on Tau levels at different exposure times. Values are shown as $\pm \mathrm{SEM}, n=3$. 
Lentivirus (-) at DIV1 and incubated for 1 week. An immunocomplex was brought down by PHF-1 that included BAG2-Flag, Hsp70 and small amounts of CHIP (Fig. 2A). In the absence of the BAG2-Flag infection, the amount of coimmunoprecipitated Hsp70 was markedly reduced and the amount of coimmunoprecipitated CHIP remained negligible. In the reverse immunoprecipitation, the M2 Flag antibody brought down PHF-1 Tau, Hsp70, and interestingly considerably more CHIP (Fig. 2A). Together, these data point to the existence of an immuno-complex that contains PHF-1 Tau, BAG2, Hsp70, and small amounts of CHIP, as well as BAG2-CHIP complexes that do not contain Tau, but may serve to inhibit CHIP-mediated ubiquitination of Tau.

BAG2, in its role as a CHIP inhibitor (Arndt et al., 2005), prevents the ubiquitination of Tau and would be expected to inhibit ubiquitin-dependent targeting of Tau to the proteasome. If CHIP-mediated Tau degradation were the only Tau degradation pathway, one would expect to see an increase of Tau in BAG2-expressing cells. Instead, the observed decrease in Tau levels suggested that BAG2 may be shuttling Tau to a ubiquitin-independent pathway. To determine whether ubiquitin was involved in BAG2-mediated Tau degradation, blots from the COS-7 cells were also probed for ubiquitinated Tau. High molecular weight Tau bands corresponding to ubiquitinated Tau were confirmed by immunoprecipitating the cell lysate with TAU-5 antibody and labeling the precipitant with ubiquitin antibody. A band of exactly the same size as the high molecular weight Tau-immunoreactive band was observed. Coexpression with BAG2 markedly reduced the ubiquitinated Tau (Fig. 2 B).

To prove that the Tau degradation enhanced by BAG2 was independent of ubiquitin, we blocked ubiquitin-mediated degradation with the K48R ubiquitin mutant and the Ub-KO mutant (see Materials and Methods). Neither mutant blocked BAG2mediated degradation (Fig. 2C), thereby providing strong evidence that the BAG2-degradation pathway was independent of ubiquitin. Interestingly, both mutants not only failed to increase the amount of Tau, they actually enhanced Tau degradation compared with BAG2 alone. This increased Tau degradation is probably due to caspase activation (see supplemental data, available at www.jneurosci.org as supplemental material). To test this possibility, cells expressing Tau and BAG2 were treated with the caspase inhibitor Z-VAD, which inhibited the Tau clearance effects of BAG2 in the presence of the K48R mutant (data not shown). Caspase-3/7 cleavage of Tau occurs in AD (Berry et al., 2003; Ding et al., 2006), and as suggested here, this Tau degradation route may result from blocking Ub-dependent degradation.

Ubiquitin-independent pathways may or may not be independent of the proteasome (Chen et al., 2004; Li et al., 2007). To resolve these possibilities cells expressing BAG2 and Tau were treated with lactacystin to inhibit the proteasome (Fig. 2D). Lactacystin blocked the Tau-clearance effects of BAG2. This treatment increased both phosphorylated and total Tau, but with a greater effect on phospho-Tau. This increase in Tau occurred despite the fact that blockade of proteasomal function by lactacystin can activate caspase (Lang-Rollin et al., 2004). However, Z-VAD did not block the effects of BAG2 on Tau clearance (Fig. $2 D)$, suggesting that in the presence of otherwise intact degradation pathways, Tau is not triaged to a caspase pathway by BAG2 in a major way. These data suggest that the mechanism by which BAG2 promotes Tau degradation is mediated by the proteasome in a ubiquitin-independent manner.

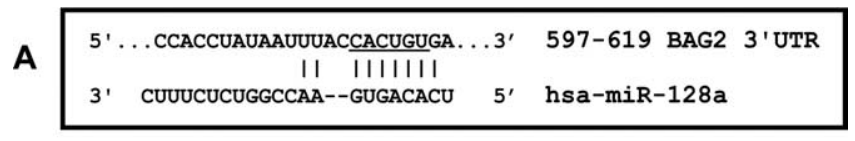

B

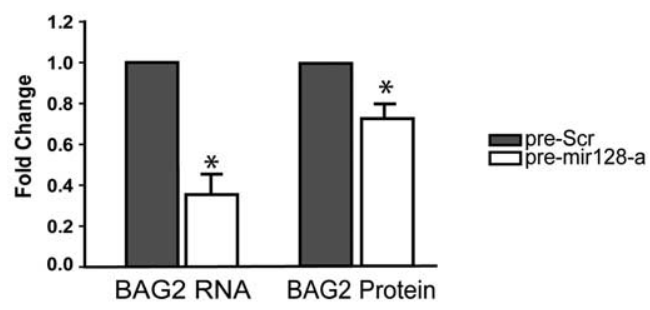

C

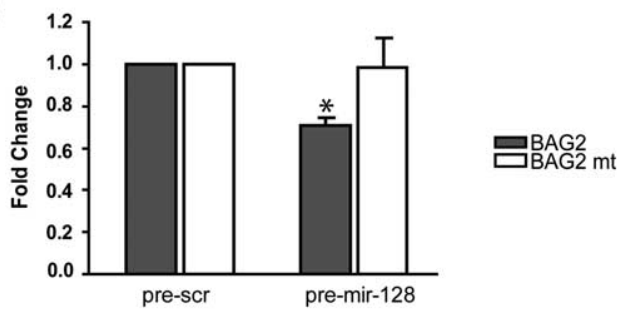

D

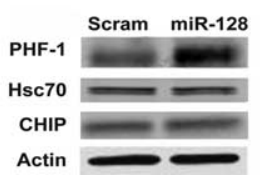

Figure 3. BAG2 is regulated by miR-128a. A, miR-128a predicted target site on BAG23'UTR. $B$, Reduction of BAG2 mRNA in response to miR-128a in neurons. Real-time PCR analysis of BAG2 mRNA showed a significant decrease in BAG2 transcript levels in response to $75 \mathrm{~nm}$ premiR-128a addition compared with scrambled ( ${ }^{*} p<0.05$ ). RNA was harvested $72 \mathrm{~h}$ after transfection of DIV 5 neurons. Reduction of BAG2 protein levels in response to miR-128a treatment of COS-7 cells. COS-7 cells were cotransfected with a FLAG-BAG2 expressing vector and pre-mir128a or a scrambled negative control. Lysates were harvested $24 \mathrm{~h}$ after transfection. Values were normalized to actin $\left({ }^{*} p<0.05\right)$. C, Dual Luciferase reporter assay performed in HeLa cells using Firefly luciferase constructs fused to a 60 bp sequence from the BAG2 $3^{\prime}$ UTR containing the miR-128a site (BAG2) or a mutant with three altered bases at the seed region (BAG2 mt, underlined in A). Constructs were transfected in conjunction with pre-miR-128a or pre-mir-scram and luciferase activity was normalized to Renilla expression $\left({ }^{*} p<0.05\right)$. D, Twofold increase of PHF-Tau in miR-128a transfected neurons. DIV 5 neurons were transfected with pre-mir-128a (250 nM) and a negative control Scrambled ( $250 \mathrm{~nm}$ ). Lysates were harvested $2 \mathrm{~d}$ after transfection. Immunoblot detection of PHF-1, Hsp70, CHIP and actin. The change in PHF-1 tau was normalized to actin and was statistically significant $\left({ }^{*} p<0.05\right)$ by the Student $t$ test, $n=3$.

\section{Physiological regulation of BAG2 by miR-128a validates the BAG2/Tau pathway}

The microRNA, miR-128a, is predicted by TargetScan and PicTar to target the BAG2 3' untranslated region (BAG2 3'UTR) with a $7 \mathrm{nt}$ complementarity in the seed region (Fig. 3A) (Krek et al., 2005; Grimson et al., 2007). The site, located between 597 and 619 bp of the human BAG2 3'UTR, is conserved in mouse, rat and dog. DIV 5 neurons were treated with pre-miR-128a and RNA was harvested at day 8. BAG2 RNA levels fell fourfold upon addition of mir-128a compared with a scrambled control (Fig. $3 B$ ). The addition of pre-miR-128a to COS-7 cells transfected with FLAG-BAG2 also decreased the protein levels of the fusion protein (Fig. $3 B$ ). To test whether the regulation of BAG2 by mir-128a is direct, we fused a 60 bp sequence of the BAG2 $3^{\prime} \mathrm{UTR}$ containing the mir-128a site downstream of a luciferase gene. miR-128a addition decreased the luciferase levels of the reporter by $32 \%$, whereas the $3^{\prime}$ UTR construct with a mutated seed region rescued the repression (Fig. $3 C$ ). Finally, treatment with miR128a in DIV 5 neurons induced a twofold increase in PHF Tau 

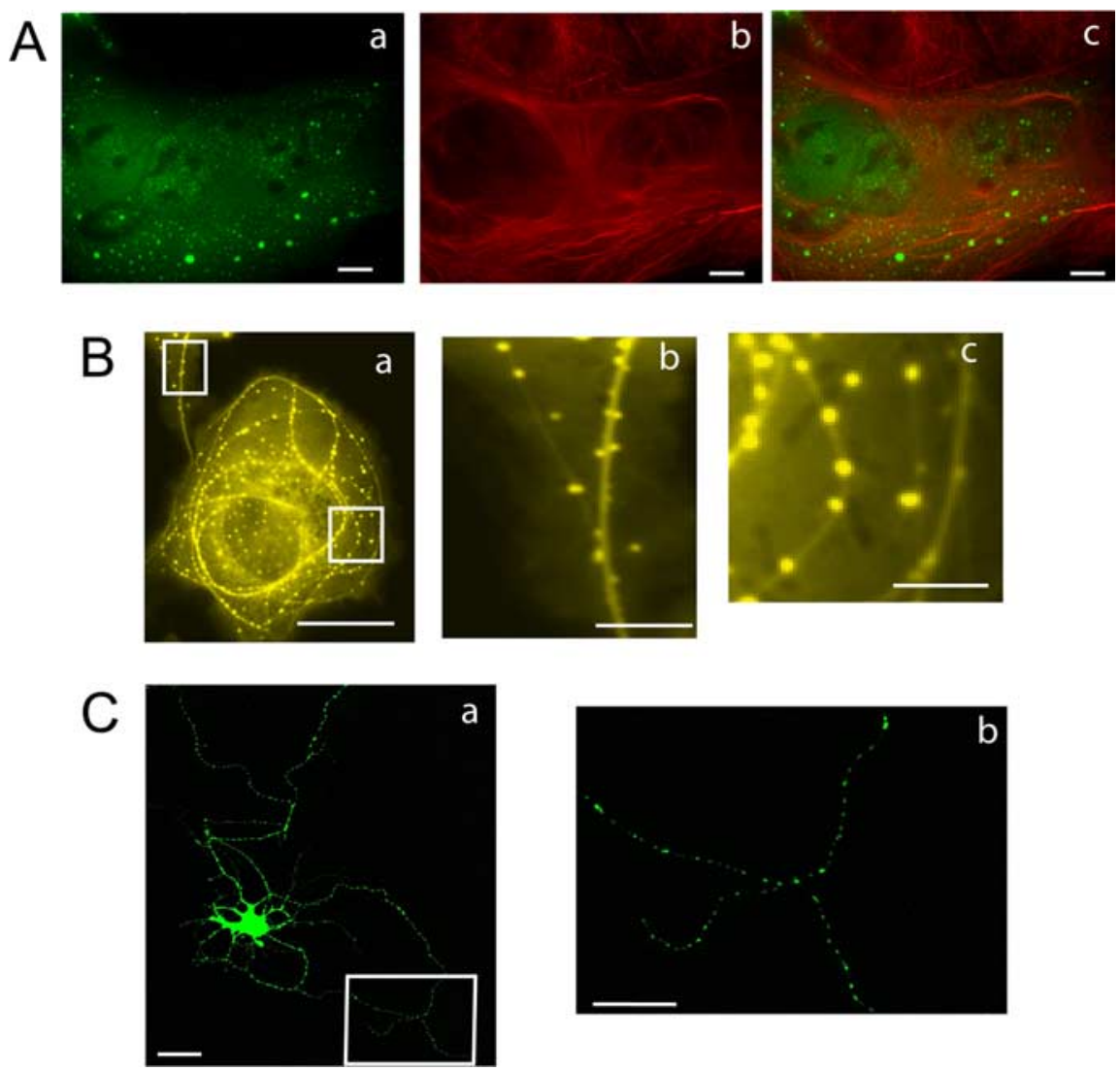

Figure 4. BAG2 colocalizes with Tau. $\boldsymbol{A}$, Quantification of BAG2/Tau colocalization. BAG2 YFP expression in COS7 is punctate (green, $\boldsymbol{a}) . \alpha$-Tubulin counterstaining with Alexa-594 (red, $\boldsymbol{b}$ ) antibody shows a high degree of colocalization of BAG2 with microtubules (c): in the presence of Tau, the number of puncta that colocalize with microtubules increases twofold from 35 to $70 \%$ (scale bar, $10 \mu \mathrm{m}) . \boldsymbol{B},(\boldsymbol{a}-\boldsymbol{c})$ Cotransfection of pEYFP-C1 BAG2 and Tau pEYFP-C1 into COS7 cells result in BAG2 puncta that align with microtubules $24 \mathrm{~h}$ after transfection. eYFP fluorescent markers provided a proportionate signal balance for the two proteins. Images $\boldsymbol{a}$ and $\boldsymbol{b}$ correspond to the red boxed regions in $\boldsymbol{b}$. Scale bars: $(\boldsymbol{b}) 10 \mu \mathrm{m} ;(\boldsymbol{a}, \boldsymbol{c}) 2 \mu \mathrm{m}$. $\boldsymbol{C}$, In rat hippocampal neurons, BAG2 distributes with microtubules in a punctate pattern. pEYFP-C1 BAG2 was transfected into primary neurons at DIV 5 and visualized $24 \mathrm{~h}$ later. The BAG2 signal extends into both axons and dendrites. Scale bars: (a) $10 \mu \mathrm{m} ;(\boldsymbol{b}) 5 \mu \mathrm{m}$.

$(p<0.05)$, detected by PHF-1 antibody (Fig. 3D). Upregulation of miR-128a had no effect on the protein levels of Hsp70 or CHIP, suggesting that the increase in PHF Tau is not due to changes in CHIP or Hsp70 (Fig. 3D). By deregulating the control pathway through miR-128a, we independently validated the relationship between BAG2 and Tau.

\section{BAG2/Hsp70 tethering to the microtubule}

Transfection of BAG2 into COS-7 cells resulted in discrete BAG2 puncta at $15-24 \mathrm{~h}$ after transfection (Fig. $4 A$ ). $28 \pm 11 \%$ of these puncta colocalized with microtubules. Coexpression of Tau and BAG2 showed a qualitatively similar pattern (Fig. 4B); however the proportion of BAG2 puncta that colocalize with microtubules increased to $72 \pm 11 \%$. Similarly, BAG2 codistributes with microtubules in neurons and appeared as puncta in both axons and dendrites (Fig. 4C).

To observe the dynamic relationship between BAG2, Tau, and the microtubules, COS-7 cells were transfected with a BAG2-YFP plasmid and in some cases with a Tau-CFP plasmid. BAG2 puncta positions were tracked both with and without Tau at 250 ms intervals. Most of the puncta moved within $1 \mu \mathrm{m}$ span of their starting point, when observed over a $75 \mathrm{~s}$ acquisition period (Fig. $5 A-C$ ), a region compatible with their diffusion coefficients, $D$, and the observation time (see supplemental Movie, available at www.jneurosci.org as supplemental material). With Tau, we measured $D=0.044 \pm 0.025 \mu \mathrm{m}^{2} \cdot \mathrm{s}^{-1}$ and without Tau $D=0.096 \pm 0.060 \mu \mathrm{m}^{2}$. $\mathrm{s}^{-1}$ (Fig. 5D).

To characterize BAG2 mobility in more detail, we measured the angular distribution of puncta displacements between every three successive puncta positions taken at $250 \mathrm{~ms}$ intervals. Near $180^{\circ}$, a significant departure occurred from a flat distribution (Fig. $5 E$ ). While a flat distribution is the signature of a Brownian motion, the enrichment near $180^{\circ}$ indicated a propensity for puncta to return to their approximate starting point after the second 250 $\mathrm{ms}$ step as well as a linear bias in the movement. The most likely explanation for the $180^{\circ}$ bias is that puncta are spring-like tethered and, together with the colocalization, the tethering is probably to a site on the microtubule. The spread of the angle distribution $\sim 180^{\circ}$ can be linked to the diffusion coefficient $D$ of the puncta and the length of the tether (see supplemental data, available at www.jneurosci.org as supplemental material). For any given $D$, the longer the tether, the wider the spread. Tau widened the spread of angles compared with cells without Tau suggesting that the leash tethering BAG2 puncta has increased in length. In the absence of Tau, that length approximated the derived size of the puncta, suggesting that puncta lie very near the site where the leash is bound, probably the microtubule. However, in the presence of Tau, the leash was 5 times longer and measured $60 \pm 20 \mathrm{~nm}$, a value comparable with the size of Tau (Ruben et al., 1991).

An analysis of reversal rates further supported BAG2 tethering. The probability of observing a reversal after a given number $i$ of forward steps was much higher for the first step and then abruptly fell to random (Fig. $5 F$ ). Thus, for $i=1$ (one step), reversal events were overrepresented, and for $i>1$ the motion was indistinguishable from Brownian. This finding suggests that BAG2 puncta bear two mobility behaviors: unbiased Brownian motion with a probability $P_{\text {brown }}$ and tethering with a probability $P_{\text {tether, }}$, with the relation $P_{\text {brown }}+P_{\text {tether }}=1$. Taking all $i>1$ steps, we determined those steps which were attributable to Brownian motion and computed the duration that each punctum spent moving in Brownian motion. When cumulated over all the puncta, we computed the totality of the tethering events as a ratio of the cumulated observation duration. Tau increased the fraction of time over which BAG2 puncta were tethered, $P_{\text {tether }}$, from $35.8 \pm 0.5 \%$ to $68.0 \pm 0.6 \%$ (see supplemental data, available at www.jneurosci.org as supplemental material).

BAG2 is known to bind to Hsp70 which might be the tethering element. To test this hypothesis, we inhibited Hsp70 using KNK437 (Yokota et al., 2000). Interestingly, this treatment induced a dramatic loss of puncta during tracking due to their enhanced diffusion outside the focal plane. For example, upon Hsp70 inhibition, tracking failed in almost $98 \%$ of the cases upon 40 frames, this figure was only 64\% with normal levels (Fig. 6A). The dependence of BAG2 localization on Hsp70 was also sug- 

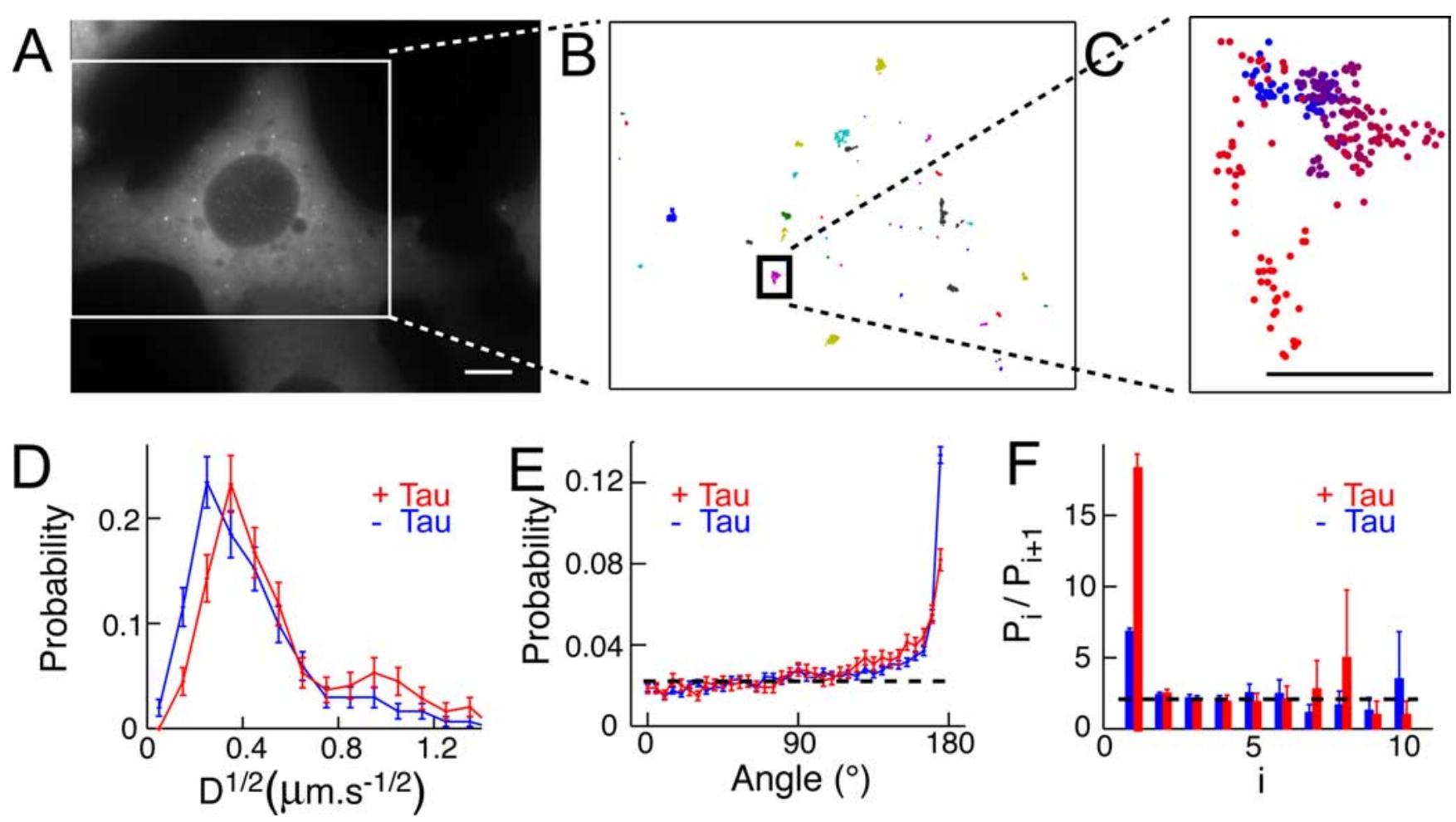

Figure 5. BAG2 puncta in COS-7 cells. A, YFP was imaged $20 \mathrm{~h}$ after transfection of Tau and BAG2-YFP (scale bar, $10 \mu \mathrm{m}$ ). $\boldsymbol{B}$, Successive positions of the puncta (imaging frequency: $4 \mathrm{~Hz}$, $\boldsymbol{B}$ corresponds to the region within the white box in $A$ ): each color represents one punctum. 0 ver the $75 \mathrm{~s}$ acquisition duration, displacement for most puncta is $<1 \mu \mathrm{m}$. $C$, Successive positions of a single punctum within the black rectangle. Positions are determined every $250 \mathrm{~ms}$ for 300 frames. Point color varies from blue to red with time as follows: color $(i)=$ blue $\times(1-i / 300)+$ red $\times$ $i / 300$; $i$ is frame number with $0 \leq i \leq 300$ (scale bar, $1 \mu \mathrm{m}$ ). D, Distribution of BAG2 puncta diffusion coefficients (red with Tau, blue without Tau). $\boldsymbol{E}$, Mobility bias toward linear reversals. Angular distribution of puncta mobility departs significantly, near $180^{\circ}$, from a flat distribution, which would be expected in the case of Brownian motion only (black dash line), suggesting a bias toward a linear motion, most likely along the microtubule (red with Tau, blue without Tau). $\boldsymbol{F}$, Ratio of two successive probabilities to reverse direction after $(i+1) \times 250 \mathrm{~ms}$ (red with Tau, blue without Tau). BAG2 puncta have an extremely high reversal rate when observed for up to $500 \mathrm{~ms}$. When apparent forward motion lasted $>500 \mathrm{~ms}(i=2)$, the ratio of two successive reversal probabilities was not significantly different from two (dash line), a value expected for a Brownian motion. Error bars represent SEM.

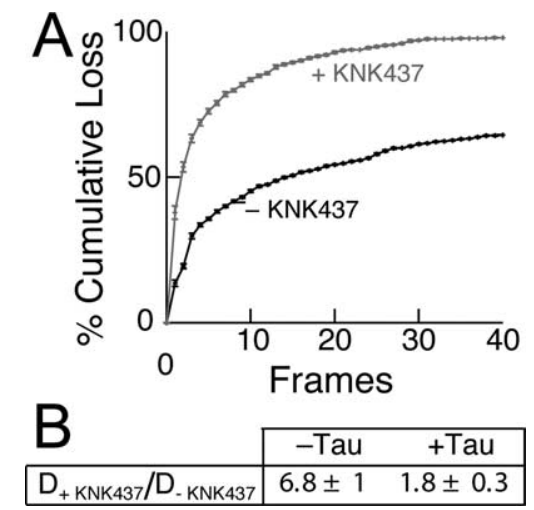

Figure 6. Changes of BAG2 mobility properties upon Hsp70 inhibition in COS-7 cells. $\boldsymbol{A}$, Cumulative loss of puncta with tracking duration. For each punctum, we determined the number of frames during which the punctum can be successfully tracked. Inhibition of Hsp70 using KNK437 induced a highly significant loss of puncta from the focal plane due to their enhanced diffusion. (black: - KNK437; gray: + KNK437). Error bars represent SEM. $\boldsymbol{B}$, Relative change of the diffusion coefficient $D$ upon treatment with KNK437. KNK437 induced an increase of $D$ both with and without Tau.

gested by the change of diffusion coefficients, $D$. Upon inhibition of Hsp70 with KNK437, these values were $D=0.23 \pm 0.12 \mu \mathrm{m}^{2}$ $\cdot \mathrm{s}^{-1}$ with Tau and $D=0.31 \pm 0.14 \mu \mathrm{m}^{2} \cdot \mathrm{s}^{-1}$ without Tau (see supplemental data, available at www.jneurosci.org as supplemental material). After correcting for changes in puncta size, KNK437 treatment resulted in a twofold increase in $D$ in the presence of Tau and a sevenfold increase in the absence of Tau (Fig. 6B). These data suggest that regardless of Tau, the Hsp70BAG2 complex is restrained from diffusing by a putative tether. When Hsp70 was inhibited with KNK437, the probability of a reversal after a single forward step decreased (see supplemental data, available at www.jneurosci.org as supplemental material). In the absence of Tau, $P_{\text {tether }}$ dropped modestly to $31.1 \pm 2.6 \%$. In the presence of Tau this percentage dropped more dramatically to $46.5 \pm 1.5 \%$. This effect of Hsp70 inhibition on tethering is consistent with the increased diffusion coefficient of BAG2 puncta after KNK437 treatment.

\section{Discussion}

The cochaperone, BAG2 markedly increased Tau degradation and selectively reduced the levels of Sarkosyl insoluble Tau. The acquisition of Sarkosyl insolubility is believed to represent a step in the misfolding of Tau that leads to inclusions. Among the regulatory elements that control BAG2 is the microRNA, miR128a. Upon miR-128a treatment, phosphorylated forms of Tau were more abundant consistent with a functional role for this miRNA as a BAG2 regulator. miR-128a is upregulated in Alzheimer's disease (Lukiw, 2007) and the resulting decreased strength of BAG2-mediated Tau degradation pathways could confer risk for neurodegeneration. BAG2 enhanced degradation of Tau was not impeded by dominant negative Ubiquitin mutants; however, its degradation was impeded by lactacystin, an inhibitor of the proteasome. Together these findings suggest that BAG2 mediates ubiquitin independent degradation of Tau through the protea- 


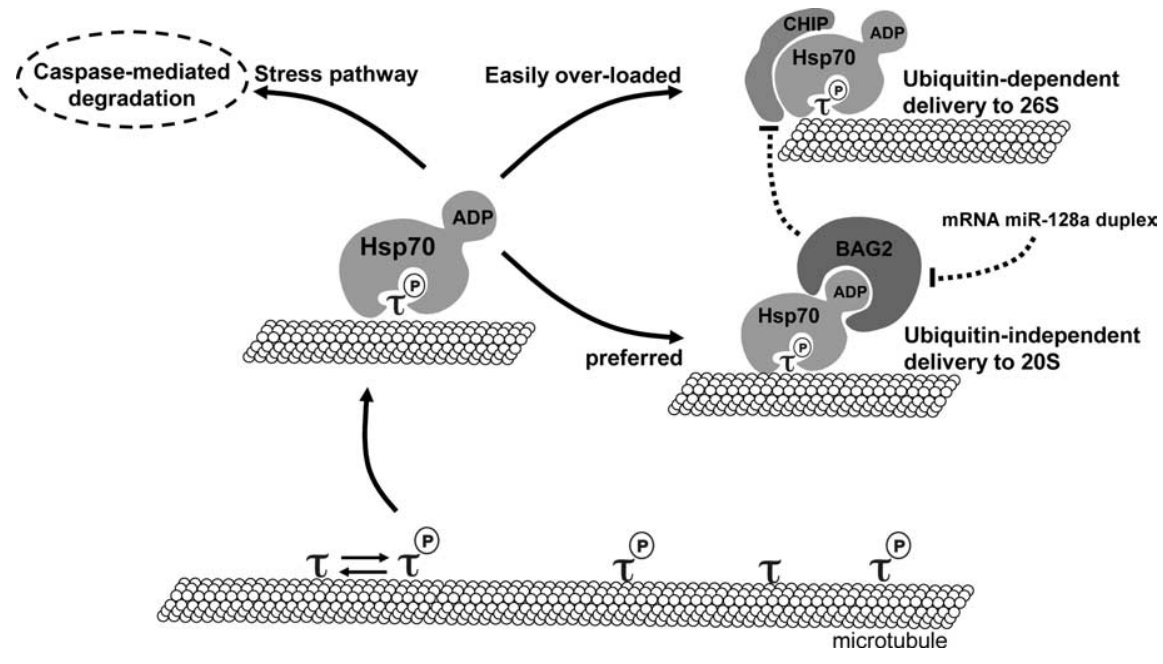

Figure 7. Model for the role of BAG2 in the regulation of Tau clearance.

compared with the reported Tau velocities. Thus, BAG2 captures and tethers Tau and possibly makes it available for delivery to the proteasome (Fig. 7).

Given the nanomolar affinity of Tau protein for microtubules (Makrides et al., $2004)$ and its cellular abundance $(\mu \mathrm{M})$, a large fraction of Tau species is found on the microtubules. Thus, positioning the triage decision at the microtubule is a highly expedient strategy. By reducing the relative abundance of phospho-Tau species, BAG2 may also reduce the tendency toward neurofibrillary tangle formation by specifically limiting the pool of Tau that has lower affinity for microtubules and are prone to aggregate. BAG2 can associate with microtubules in the absence of Tau (Fig. 4A) (Gache et al., 2005). The addition of Tau to a cell results in further recruitment of BAG2 to the

some. A pathway such as that outlined here may allow access to internal folding defects, and thereby effectively degrade natively disordered substrates at internal peptide bonds (Weinreb et al., 1996). Examples of proteins degraded in this pathway are the cyclin-dependent kinase inhibitor p $21^{\text {cipl }}$ (Sheaff et al., 2000; Touitou et al., 2001), translation initiation factors eIF4G and eIF3a (Baugh and Pilipenko, 2004), p53 (Asher et al., 2002), and $\alpha$-synuclein (Tofaris et al., 2001; Liu et al., 2003). Tau is also natively unfolded and evidence of Ubiquitin-independent Tau degradation has been published (Cardozo and Michaud, 2002; David et al., 2002).

The complexity of Tau degradation pathways might be viewed as a hierarchy. Degradation of misfolded Tau occurs preferentially in a ubiquitin-independent manner. Under various conditions Tau can get shunted to a ubiquitin-dependent pathway, but this pathway may be less efficient as suggested by the finding that overexpressed CHIP did not reduce Tau levels (Dickey et al., 2006). Thus, CHIP mediated ubiquitin-dependent degradation may become more readily saturated than the BAG2 mediated ubiquitin-independent degradation. Under these circumstances, Tau is ubiquitinated, and based on the longstanding observation that Tau inclusions are ubiquitinated, Tau ubiquitination may make Tau prone to aggregation. While inclusions may represent a temporizing protective measure to create an inert body of nondegradable protein, a riskier strategy to rid the cell of Tau is caspase activation. When the proteasome becomes inhibited in the presence of inclusions, caspases are activated (Bence et al., 2001), and Tau is an efficient substrate for caspase as well as further downstream enzymes such as calpains (Johnson, 2006; Park et al., 2007). However, caspases turn off protective pathways and lead to cellular destruction. The findings here suggest that Tau may become a caspase substrate only under stressful conditions that make proteasomal degradation pathways inaccessible.

BAG2 undergoes two types of mobility: spring-like tethering and Brownian excursions between tethering events. Previously, Tau was reported to move along the axon at rates consistent with slow transport $(0.1-8 \mathrm{~mm} / \mathrm{d}$, or $0.001-0.08 \mu \mathrm{m} / \mathrm{s}$ ) (Mercken et al., 1995). More recently, unexpectedly fast diffusion-like movements of Tau at $\sim 1 \mu \mathrm{m} / \mathrm{s}$ have been observed along microtubules that were explained by the highly dynamic interaction between Tau and the microtubule (Konzack et al., 2007). However the increased number of BAG2 tethering events induced by Tau indicates that the BAG2/Hsp70/Tau complex is relatively static microtubules. Thus, BAG2 targets misfolded Tau on the microtubules, a pool of Tau that has been considered a source of Tau filaments (Ackmann et al., 2000; Lu and Kosik, 2001).

Inhibition of Hsp70 using KNK437 resulted in a significant decrease of BAG2 tethering suggesting that Hsp70 plays a key role in anchoring BAG2 on the microtubule. Hsp70 is also involved in Tau triage by binding through its ATPase domain to the C-terminal region of BAG family members (Takayama et al., 1997; Zeiner et al., 1997). Binding in this region gives rise to the chaperone inhibitory properties of BAG family members by preventing the ATP-dependent release of the Hsp70-associated substrate (Bimston et al., 1998). The effect of this complex formation may maintain the substrate in a soluble non-native state at the point of triage. The recent report that BAG1 overexpression impedes ubiquitin-independent Tau degradation (Elliott et al., 2007) can be explained in the light of our results: BAG1 and BAG2 compete for the same binding site, and are pivotally positioned to determine alternative fates of misfolded Tau, i.e., either proteolysis in a ubiquitin independent or dependent manner. The E3 ligase CHIP can also mediate Tau degradation by interacting with Hsp70. By inhibiting CHIP (Qian et al., 2006), BAG2 directs the Hsp70-Tau complex away from ubiquitination. In contrast to BAG1, the lack of an N-terminal ubiquitin-like domain in BAG2 makes it well suited for ubiquitin-independent delivery of substrates to the proteasome.

In summary, our data suggest that BAG2 plays a critical role in shuttling a pool of microtubule-associated Tau, prone to misfolding, to a ubiquitin-independent pathway for degradation. Because phosphorylation controls both Tau binding to the microtubule and its tendency to misfold, the localization of this degradation pathway to the microtubule is well positioned to divert Tau from ubiquitination, and ubiquitin-dependent delivery to the proteasome, and possibly aggregate formation. Hsp70 plays a key role in Tau triage. We hypothesize that Tau triage decisions are related to the relative concentrations of the cochaperones, BAG1, BAG2, and CHIP for competitive binding to Hsp70. Routing Tau to a less efficient degradation pathway, will lead to excess misfolded Tau and ultimately the formation of neurofibrillary tangles.

\section{References}

Ackmann M, Wiech H, Mandelkow E (2000) Nonsaturable binding indicates clustering of tau on the microtubule surface in a paired helical filament-like conformation. J Biol Chem 275:30335-30343. 
Alberti S, Demand J, Esser C, Emmerich N, Schild H, Hohfeld J (2002) Ubiquitylation of BAG-1 suggests a novel regulatory mechanism during the sorting of chaperone substrates to the proteasome. J Biol Chem 277:45920-45927.

Arndt V, Daniel C, Nastainczyk W, Alberti S, Höhfeld J (2005) BAG-2 acts as an inhibitor of the chaperone-associated ubiquitin ligase CHIP. Mol Biol Cell 16:5891-5900.

Asher G, Lotem J, Kama R, Sachs L, Shaul Y (2002) NQO1 stabilizes p53 through a distinct pathway. Proc Natl Acad Sci U S A 99:3099-3104.

Ballatore C, Lee VM, Trojanowski JQ (2007) Tau-mediated neurodegeneration in Alzheimer's disease and related disorders. Nat Rev Neurosci 8:663-672.

Baugh JM, Pilipenko EV (2004) 20S proteasome differentially alters translation of different mRNAs via the cleavage of eIF4F and eIF3. Mol Cell 16:575-586.

Bence NF, Sampat RM, Kopito RR (2001) Impairment of the ubiquitinproteasome system by protein aggregation. Science 292:1552-1555.

Berger Z, Ravikumar B, Menzies FM, Oroz LG, Underwood BR, Pangalos MN, Schmitt I, Wullner U, Evert BO, O’Kane CJ, Rubinsztein DC (2006) Rapamycin alleviates toxicity of different aggregate-prone proteins. Hum Mol Genet 15:433-442.

Bergink S, Salomons FA, Hoogstraten D, Groothuis TA, de Waard H, Wu J, Yuan L, Citterio E, Houtsmuller AB, Neefjes J, Hoeijmakers JH, Vermeulen W, Dantuma NP (2006) DNA damage triggers nucleotide excision repair-dependent monoubiquitylation of histone H2A. Genes Dev 20:1343-1352.

Berry RW, Abraha A, Lagalwar S, LaPointe N, Gamblin TC, Cryns VL, Binder LI (2003) Inhibition of tau polymerization by its carboxy-terminal caspase cleavage fragment. Biochemistry 42:8325-8331.

Bimston D, Song J, Winchester D, Takayama S, Reed JC, Morimoto RI (1998) BAG-1, a negative regulator of Hsp70 chaperone activity, uncouples nucleotide hydrolysis from substrate release. EMBO J 17:6871-6878.

Cardozo C, Michaud C (2002) Proteasome-mediated degradation of tau proteins occurs independently of the chymotrypsin-like activity by a nonprocessive pathway. Arch Biochem Biophys 408:103-110.

Chen X, Chi Y, Bloecher A, Aebersold R, Clurman BE, Roberts JM (2004) $\mathrm{N}$-acetylation and ubiquitin-independent proteasomal degradation of p21(Cip1). Mol Cell 16:839-847.

Cho JH, Johnson GV (2004) Glycogen synthase kinase 3 beta induces caspase-cleaved tau aggregation in situ. J Biol Chem 279:54716-54723.

Cripps D, Thomas SN, Jeng Y, Yang F, Davies P, Yang AJ (2006) Alzheimer disease-specific conformation of hyperphosphorylated paired helical filament-Tau is polyubiquitinated through Lys-48, Lys-11, and Lys-6 ubiquitin conjugation. J Biol Chem 281:10825-10838.

Dantuma NP, Groothuis TA, Salomons FA, Neefjes J (2006) A dynamic ubiquitin equilibrium couples proteasomal activity to chromatin remodeling. J Cell Biol 173:19-26.

David DC, Layfield R, Serpell L, Narain Y, Goedert M, Spillantini MG (2002) Proteasomal degradation of tau protein. J Neurochem 83:176-185.

Demand J, Alberti S, Patterson C, Höhfeld J (2001) Cooperation of a ubiquitin domain protein and an E3 ubiquitin ligase during chaperone/proteasome coupling. Curr Biol 11:1569-1577.

DeTure M, Ko LW, Easson C, Yen SH (2002) Tau assembly in inducible transfectants expressing wild-type or FTDP-17 tau. Am J Pathol 161:1711-1722.

Dickey CA, Yue M, Lin WL, Dickson DW, Dunmore JH, Lee WC, Zehr C, West G, Cao S, Clark AM, Caldwell GA, Caldwell KA, Eckman C, Patterson C, Hutton M, Petrucelli L (2006) Deletion of the ubiquitin ligase CHIP leads to the accumulation, but not the aggregation, of both endogenous phospho- and caspase-3-cleaved tau species. J Neurosci 26:6985-6996.

Dickey CA, Kamal A, Lundgren K, Klosak N, Bailey RM, Dunmore J, Ash P, Shoraka S, Zlatkovic J, Eckman CB, Patterson C, Dickson DW, Nahman NS Jr, Hutton M, Burrows F, Petrucelli L (2007) The high-affinity HSP90-CHIP complex recognizes and selectively degrades phosphorylated tau client proteins. J Clin Invest 117:648-658.

Ding H, Matthews TA, Johnson GV (2006) Site-specific phosphorylation and caspase cleavage differentially impact tau-microtubule interactions and tau aggregation. J Biol Chem 281:19107-19114.

Elliott E, Tsvetkov P, Ginzburg I (2007) BAG-1 associates with Hsc70.Tau complex and regulates the proteasomal degradation of Tau protein. J Biol Chem 282:37276-37284.
Fenteany G, Schreiber SL (1998) Lactacystin, proteasome function, and cell fate. J Biol Chem 273:8545-8548.

Gache V, Louwagie M, Garin J, Caudron N, Lafanechere L, Valiron O (2005) Identification of proteins binding the native tubulin dimer. Biochem Biophys Res Commun 327:35-42.

Grimson A, Farh KK, Johnston WK, Garrett-Engele P, Lim LP, Bartel DP (2007) MicroRNA targeting specificity in mammals: determinants beyond seed pairing. Mol Cell 27:91-105.

Heale BS, Soifer HS, Bowers C, Rossi JJ (2005) siRNA target site secondary structure predictions using local stable substructures. Nucleic Acids Res 33:e30.

Ii K, Ito H, Tanaka K, Hirano A (1997) Immunocytochemical colocalization of the proteasome in ubiquitinated structures in neurodegenerative diseases and the elderly. J Neuropathol Exp Neurol 56:125-131.

Iqbal K, Grundke-Iqbal I (1991) Ubiquitination and abnormal phosphorylation of paired helical filaments in Alzheimer's disease. Mol Neurobiol 5:399-410

Jenkins SM, Zinnerman M, Garner C, Johnson GV (2000) Modulation of tau phosphorylation and intracellular localization by cellular stress. Biochem J 345:263-270.

Jicha GA, O’Donnell A, Weaver C, Angeletti R, Davies P (1999) Hierarchical phosphorylation of recombinant tau by the paired-helical filamentassociated protein kinase is dependent on cyclic AMP-dependent protein kinase. J Neurochem 72:214-224.

Johnson GV (2006) Tau phosphorylation and proteolysis: insights and perspectives. J Alzheimers Dis 9:243-250.

Konzack S, Thies E, Marx A, Mandelkow EM, Mandelkow E (2007) Swimming against the tide: mobility of the microtubule-associated protein tau in neurons. J Neurosci 27:9916-9927.

Krek A, Grün D, Poy MN, Wolf R, Rosenberg L, Epstein EJ, MacMenamin P, da Piedade I, Gunsalus KC, Stoffel M, Rajewsky N (2005) Combinatorial microRNA target predictions. Nat Genet 37:495-500.

Lang-Rollin I, Vekrellis K, Wang Q, Rideout HJ, Stefanis L (2004) Application of proteasomal inhibitors to mouse sympathetic neurons activates the intrinsic apoptotic pathway. J Neurochem 90:1511-1520.

Li X, Amazit L, Long W, Lonard DM, Monaco JJ, O’Malley BW (2007) Ubiquitin- and ATP-independent proteolytic turnover of p21 by the REGgamma-proteasome pathway. Mol Cell 26:831-842.

Liu CW, Corboy MJ, DeMartino GN, Thomas PJ (2003) Endoproteolytic activity of the proteasome. Science 299:408-411.

Lu M, Kosik KS (2001) Competition for microtubule-binding with dual expression of tau missense and splice isoforms. Mol Biol Cell 12:171-184.

Lüders J, Demand J, Höhfeld J (2000) The ubiquitin-related BAG-1 provides a link between the molecular chaperones Hsc70/Hsp70 and the proteasome. J Biol Chem 275:4613-4617.

Lukiw WJ (2007) Micro-RNA speciation in fetal, adult and Alzheimer's disease hippocampus. Neuroreport 18:297-300.

Makrides V, Massie MR, Feinstein SC, Lew J (2004) Evidence for two distinct binding sites for tau on microtubules. Proc Natl Acad Sci U S A 101:6746-6751.

Mercken M, Fischer I, Kosik KS, Nixon RA (1995) Three distinct axonal transport rates for tau, tubulin, and other microtubule-associated proteins: evidence for dynamic interactions of tau with microtubules in vivo. J Neurosci 15:8259-8267.

Morishima-Kawashima M, Hasegawa M, Takio K, Suzuki M, Titani K, Ihara Y (1993) Ubiquitin is conjugated with amino-terminally processed tau in paired helical filaments. Neuron 10:1151-1160.

Park SY, Tournell C, Sinjoanu RC, Ferreira A (2007) Caspase-3- and calpain-mediated tau cleavage are differentially prevented by estrogen and testosterone in beta-amyloid-treated hippocampal neurons. Neuroscience 144:119-127.

Petrucelli L, Dickson D, Kehoe K, Taylor J, Snyder H, Grover A, De Lucia M, McGowan E, Lewis J, Prihar G, Kim J, Dillmann WH, Browne SE, Hall A, Voellmy R, Tsuboi Y, Dawson TM, Wolozin B, Hardy J, Hutton M (2004) CHIP and Hsp70 regulate tau ubiquitination, degradation and aggregation. Hum Mol Genet 13:703-714.

Qian SB, McDonough H, Boellmann F, Cyr DM, Patterson C (2006) CHIPmediated stress recovery by sequential ubiquitination of substrates and Hsp70. Nature 440:551-555.

Ruben GC, Iqbal K, Grundke-Iqbal I, Wisniewski HM, Ciardelli TL, Johnson JE Jr (1991) The microtubule-associated protein tau forms a triplestranded left-hand helical polymer. J Biol Chem 266:22019-22027. 
Sahara N, Murayama M, Mizoroki T, Urushitani M, Imai Y, Takahashi R, Murata S, Tanaka K, Takashima A (2005) In vivo evidence of CHIP up-regulation attenuating tau aggregation. J Neurochem 94:1254-1263.

Sheaff RJ, Singer JD, Swanger J, Smitherman M, Roberts JM, Clurman BE (2000) Proteasomal turnover of p21Cip1 does not require p21Cip1 ubiquitination. Mol Cell 5:403-410.

Shimura H, Miura-Shimura Y, Kosik KS (2004) Binding of tau to heat shock protein 27 leads to decreased concentration of hyperphosphorylated tau and enhanced cell survival. J Biol Chem 279:17957-17962.

Takayama S, Bimston DN, Matsuzawa S, Freeman BC, Aime-Sempe C, Xie Z, Morimoto RI, Reed JC (1997) BAG-1 modulates the chaperone activity of Hsp70/Hsc70. EMBO J 16:4887-4896.

Takayama S, Xie Z, Reed JC (1999) An evolutionarily conserved family of Hsp70/Hsc70 molecular chaperone regulators. J Biol Chem 274:781-786.

Tofaris GK, Layfield R, Spillantini MG (2001) alpha-synuclein metabolism and aggregation is linked to ubiquitin-independent degradation by the proteasome. FEBS Lett 509:22-26.

Touitou R, Richardson J, Bose S, Nakanishi M, Rivett J, Allday MJ (2001) A degradation signal located in the C-terminus of $\mathrm{p} 21 \mathrm{WAF} 1 / \mathrm{CIP} 1$ is a binding site for the C8 alpha-subunit of the $20 \mathrm{~S}$ proteasome. EMBO J 20:2367-2375.

Vanmechelen E, Vanderstichele H, Davidsson P, Van Kerschaver E, Van Der Perre B, Sjögren M, Andreasen N, Blennow K (2000) Quantification of tau phosphorylated at threonine 181 in human cerebrospinal fluid: a sandwich ELISA with a synthetic phosphopeptide for standardization. Neurosci Lett 285:49-52.

Weinreb PH, Zhen W, Poon AW, Conway KA, Lansbury PT Jr (1996) NACP, a protein implicated in Alzheimer's disease and learning, is natively unfolded. Biochemistry 35:13709-13715.

Yokota S, Kitahara M, Nagata K (2000) Benzylidene lactam compound, KNK437, a novel inhibitor of acquisition of thermotolerance and heat shock protein induction in human colon carcinoma cells. Cancer Res 60:2942-2948.

Zeiner M, Gebauer M, Gehring U (1997) Mammalian protein RAP46: an interaction partner and modulator of $70 \mathrm{kDa}$ heat shock proteins. EMBO J 16:5483-5490. 